intensity resistance training led to greater improvements in muscle strength compared to moderate-intensity resistance training in patients with knee OA. This did not result in greater improvements in pain and physical functioning in the highintensity resistance group; both groups showed similar clinically important improvements. The added value of vitamin D supplementation on muscle strength in knee OA patients with vitamin D deficiency need further study.

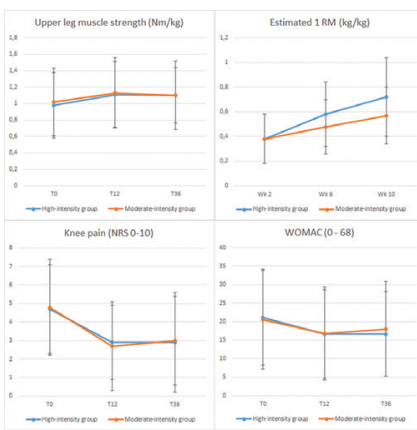

Disclosure of Interests: Arjan de Zwart: None declared, Joost Dekker: None declared, Leo D. Roorda: None declared, Martin van der Esch : None declared, Paul Lips: None declared, Natasja van Schoor: None declared, Annemieke Heijboer: None declared, Franktien Turkstra: None declared, Martijn Gerritsen Grant/ research support from: Grunenthal has sponsored the Reade Cohort, Arja Häkkinen: None declared, Kim Bennell: None declared, Martijn Steultjens: None declared, Willem Lems Speakers bureau: Amgen Inc., Merck, Eli Lilly and Pfizer, Marike van der Leeden : None declared

DOI: 10.1136/annrheumdis-2019-eular.7040

\section{OP0160-HPR DOES OCCUPATIONAL THERAPY DELAY OR REDUCE THE PROPORTION OF PATIENTS WHO RECEIVES THUMB CARPOMETACARPAL SURGERY? A RANDOMIZED CONTROLLED TRIAL}

Else Marit H. Gravås ${ }^{1}$, Nina Østerås ${ }^{1}$, Randi Nossum ${ }^{2}$, Ruth Else Mehl Eide ${ }^{3}$, Åse Klokkeide ${ }^{4}$, Karin Hoegh Matre ${ }^{3}$, Monika Olsen ${ }^{4}$, Øyvor Andreassen ${ }^{1}$, Ida Kristin Haugen ${ }^{5}$, Anne Therese Tveter ${ }^{1}$, Ingvild Kjeken ${ }^{1}$. 'Diakonhjemmet Hospital, National Advisory Unit on Rehabilitation in Rheumatology, Department of Rheumatology, Oslo, Norway; ${ }^{2}$ St. Olavs Hospital HF, Department of Rheumatology, Trondheim, Norway; ${ }^{3}$ Haukeland University Hospital/Health Bergen, Department of Rheumatology, Bergen, Norway; ${ }^{4}$ Haugesund Rheumatism Hospital, Department of Rheumatology, Haugesund, Norway, ${ }^{5}$ Diakonhjemmet Hospital, Department of Rheumatology, Oslo, Norway

Background: The 2018 update of the EULAR recommendations for the management of hand osteoarthritis (OA) states that every patient with hand OA should be offered patient education, hand exercises and provision of assistive devices. Orthoses should be considered for symptom relief in patients with thumb carpometacarpal (CMC1) OA. Surgery should only be considered when other treatment modalities have not been sufficiently effective in relieving pain (1).

Objectives: The main aim was to evaluate whether occupational therapy in the waiting period prior to surgical consultation can reduce or delay the need for surgery in $\mathrm{CMC1}$ OA. A secondary aim was to explore potential predictors for $\mathrm{CMC} 1$ surgery.

Methods: This is a multicentre parallel-group randomized controlled trial including patients referred by their general practitioner for surgical consultation due to CMC1 OA at three Norwegian departments of rheumatology. After baseline assessments, participants were randomized into an occupational therapy group or a control group and reassessed after 4, 18 and 24 months. Both groups received written and oral information on hand OA. In addition, the occupational therapy group received a hand exercise program, day and night orthoses, assistive devices and a treatment diary.

Intention-to-treat analyses were conducted fitting multivariate logistic regression analyses to compare the proportion of participants in the two groups who had received CMC1 surgery after two years. Time to surgery was examined in a Kaplan-Meier survival plot, whereas crude and adjusted logistic regression analyses were performed to investigate predictors for $\mathrm{CMC} 1$ surgery using a predefined set of covariates of demographic and clinical variables.

Results: Of 214 patients screened for eligibility, 180 (84\%) were randomized. The mean age was 63 years (SD 7.6) and 79\% were women. Eighty-four (93\%) participants in the occupational therapy group and $82(91 \%)$ in the control group completed 24 months follow-up assessments. Fifty-eight (64\%) participants in the occupation therapy group were categorized as having high treatment adherence. Twenty-two (24\%) participants in the occupational therapy group and 31 (34\%) in the control group received surgery before the two year follow-up. The odds for receiving surgery were 50 percent lower in the occupational therapy group $(p=0.10)$. Median time to surgery was 349 days (IQR 210 to 540 ) in the occupational therapy group and 267 days (IQR 181 to 406 ) in the control group ( $p=0.07$ ) Previous non-pharmacological treatment (OR 2.70, 95\% Cl 1.16 to 6.27 ) and higher motivation for surgery (OR $1.22,95 \% \mathrm{Cl} 1.07$ to 1.38 ) were significant predictors for CMC1 surgery

Conclusion: In participants receiving occupational therapy there was a clear, but not statistically significant trend towards reduction and delay in $\mathrm{CMC} 1$ surgery as compared to the control group. Previously received non-pharmacological treatment and higher motivation for surgery significantly predicted surgery. The results support that patients with $\mathrm{CMC} 1 \mathrm{OA}$ should be referred to occupational therapy before surgery is considered.

\section{REFERENCE:}

[1] Kloppenburg M, Kroon FP, Blanco FJ, Doherty M, Dziedzic KS, Greibrokk E, et al. 2018 update of the EULAR recommendations for the management of hand osteoarthritis. Ann Rheum Dis. 2019;78(1):16-24.

Disclosure of Interests: : Else Marit H. Gravås: None declared, Nina Østerås: None declared, Randi Nossum: None declared, Ruth Else Mehl Eide: None declared, Åse Klokkeide: None declared, Karin Hoegh Matre: None declared, Monika Olsen: None declared, Øyvor Andreassen: None declared, Ida Kristin Haugen Grant/research support from: ADVANCE research grant from Pfizer Consultant for: Advisory board Abbvie, Anne Therese Tveter: None declared, Ingvild Kjeken: None declared DOI: 10.1136/annrheumdis-2019-eular.986

\section{OP0161-HPR SHORT-TERM EFFECT OF OCCUPATIONAL THERAPY INTERVENTION ON HAND FUNCTION AND PAIN IN PATIENTS WITH THUMB BASE OSTEOARTHRITIS - SECONDARY ANALYSES OF A RANDOMIZED CONTROLLED TRIAL}

Anne Therese Tveter ${ }^{1}$, Randi Nossum ${ }^{2}$, Ruth Else Mehl Eide ${ }^{3}$,Åse Klokkeide ${ }^{4}$, Karin Hoegh Matre ${ }^{3}$, Monika Olsen ${ }^{4}, \varnothing_{y}$ vor Andreassen ${ }^{1}$, Nina Osteras ${ }^{1}$, Ingvild Kjeken ${ }^{1}$. ${ }^{1}$ Diakonhjemmet Hospital, National Advisory Unit on Rehabilitation in Rheumatology, Oslo, Norway, ${ }^{2}$ St.Olavs Hospital, Trondheim University Hospital, Trondheim, Norway; ${ }^{3}$ Haukeland University Hospital, Bergen, Norway; ${ }^{4}$ Haugesund Rheumatism Hospital AS, Haugesund, Norway

Background: Carpometacarpal joint osteoarthritis (CMC1 OA) is a subset of hand osteoarthritis (HOA), presenting symptoms of pain and functional limitations. No cure for OA exists, and the EULAR recommendations highlight conservative treatment as first-line treatment for patients with HOA. Although several studies have assessed the effect of different treatments on pain and function in patients with $\mathrm{HOA}$, there is a lack of high quality studies assessing these effects in patients with $\mathrm{CMC} 1 \mathrm{OA}$. A recent systematic review showed that multimodal treatments (i. e. patient education, exercises, orthoses) improved pain but not function; however, the two studies included had short intervention periods and small samples. Thus, additional studies are warranted.

Objectives: To assess the short-term effect of a 3-month multimodal occupational therapy intervention on pain and function in patients referred to surgical consultation for CMC1 OA.

Methods: This study presents secondary analyses of a multicenter randomized controlled trial. Patients referred to surgical consultation due to CMC1 OA at three departments of rheumatology in Norway between 2013 and 2015 were considered eligible if they could speak Norwegian and did not have cognitive dysfunction. During the waiting period between referral and actual surgical consultation, the 180 included patients were randomized to either usual care (information abou hand $\mathrm{OA}$ ) or multimodal occupational therapy consisting of patient education, hand exercises, orthoses and assistive devices. Patients were instructed in conducting hand exercises three times per week for 12 weeks. They were given both day and night orthoses and were instructed to use them as much as possible. They were also given five commonly used assistive devices for use at home. The patients answered questionnaires and were assessed at baseline and in conjunction with the surgical consultation (follow-up). Pain at rest and during pinch- and grip strength was self-reported on a 10-point numeric rating scale. Function was self-reported in MAP-Hand (1-4, $1=$ no problems) and QuickDASH (0-100, 0=no disability) and measured as grip- and pinch strength (\% of reference values) and range of motion (flexion deficit 2.-5.finger, palmar abduction and abduction in the CMC1). Within-group difference from baseline to follow-up was analyzed with paired sample t-test, while between-group difference at follow-up was estimated with multiple linear regression, adjusting for baseline value and time to follow-up. Due to multiple analyses of secondary outcomes, the $p$-value was set to $p<0.01$. Results: The 180 included patients' mean (SD) age was 63 (8) years and most were women (81\%). At baseline, there were no significant differences between 